\title{
Transient Superior Mesenteric Artery Syndrome and Psychogenic Vomiting
}

\author{
A Case Study in Diagnosis and Management
}

Patricia A. O'Connor, M.D.," Andrew K. Poznanski, M.D., $†$ Elva O. Poznanski, M.D.

\begin{abstract}
A well-nourished teen-age girl with psychogenic vomiting demonstrated clinical and radiographic findings of SMAS. Her upper gastrointestinal radiographic studies were normal a few weeks before and three years after the study in which radiographic findings of SMAS were present. Psychologic factors should be considered in SMAS when no condition involving inanition, immobility, abdominal compression, or coexisting abdominal disturbance can be identified.
\end{abstract}

HE SUPERIOR MESENTERIC ARTERY syndrome (SMAS), said to have been first described by Von Rokitansky in $1861,{ }^{1}$ consists of clinical findings of lower duodenal obstruction such as vomiting of bile-stained material, and indentation of the third portion of the duodenum where the superior mesenteric artery passes anteriorly (now demonstrated radiologically). A variety of theories concerning etiology, significance of findings, and therapy in this condition have been proposed. Recent reports are of cases in adults, application of radiographic diagnostic techniques, the association with other clinical and pathologic states, ${ }^{2-5}$ and surgical treatment of cases not responsive to medical management. ${ }^{2-4,6,7}$ We here describe a teenage girl with apparent psychogenic vomiting who had transient clinical and radiographic findings of SMAS. She did not have any of the physical disabilities which have been reported

\footnotetext{
* Associate Professor, Pediatrics and Communicable Diseases, University of Michigan Medical Center, Ann Arbor, Mich. 48104.

$\dagger$ Professor of Radiology.

$¥$ Assistant Professor of Psychiatry.
}

as associated with SMAS. She was treated successfully by recognition of her adjustment problems and supportive follow-up.

\section{Case Report}

S., a 13-year-old pubescent well-nourished girl, was seen at the University of Michigan Medical Center (UMMC) for recurrent vomiting between December 5, 1968 and February 7, 1969.

At the time of her first visit, she had a three-week history of morning vomiting (at times bile stained), fatigue, headaches, and dizzy spells. Physical examinations were normal except for variable mild upper abdominal tenderness. She had no fever or changes in vital signs. Persistent vomiting and ketonuria led to two admissions, December 17 to 21, and January 10 to 22. Each time she improved promptly with intravenous fluids and temporary restriction of oral feedings. During the January hospitalization an upper gastrointestinal study showed the typical radiographic findings of the superior mesenteric artery syndrome (Figs. 1a, 1b). Surgical consultants advised against surgery,

Her last clinic visit for vomiting was on February 7,1969 , when she was seen with a four-day history of vomiting. Urinalysis showed $4+$ ketones and a trace of albumin. Over a several hour period, she was given small amounts of ginger ale and water frequently and did not vomit. Ketonuria decreased to $2+$. She was discharged to continue on this regi- 

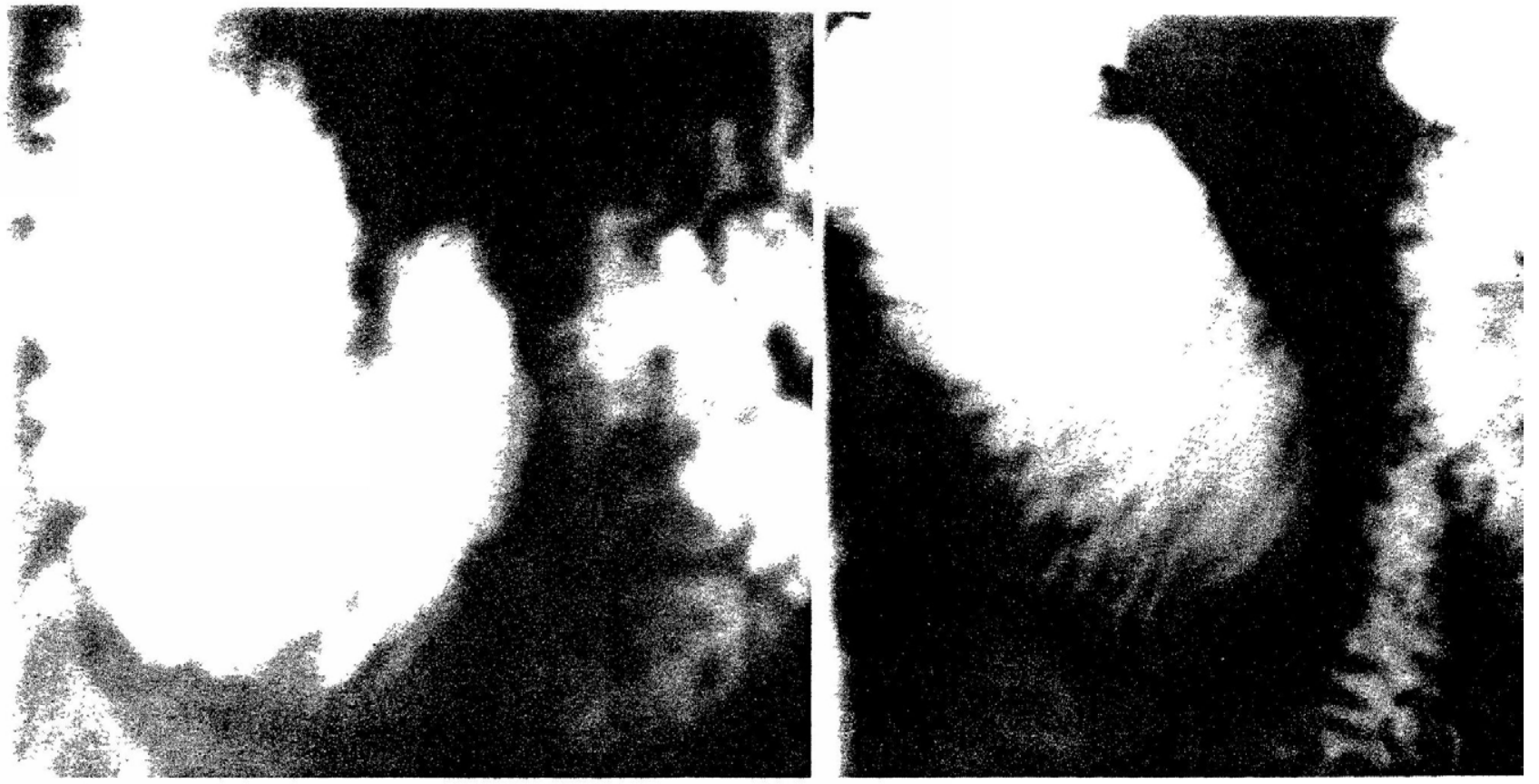

FIG. 1. Films during episodes of vomiting. la) (left) This photograph was from a conventional G-I study. 1b) (right) This photograph was during hypotonic duodenography one week later. In both films a prominent linear indentation is seen crossing the third portion of the duodenum. There was dilatation of the proximal duodenum and there was much to and fro peristalsis.

men. She was seen again on February 11,1969 and was doing well.

Psychiatric evaluations during the hospitalizations had identified problems which led to the discharge diagnosis of psychogenic vomiting. Counseling was advised but was not accepted by the parents. During the last two clinic visits, the need for her to have help in handling her feelings and the probable association between this need and her vomiting were discussed with her and her mother. Her mother seemed to accept the association but was not receptive to formal counseling.

Over the next 21 months, she was seen for minor complaints but had no significant gastrointestinal problems. On November 10, 1970, however, she was seen because of recurrent right upper quadrant pain. An upper gastrointestinal study and cholecystogram were normal without the previously demonstrated radiographic findings of the SMAS. Over the next 20 months, she was seen several times but was generally well with no significant problems.

The radiographic studies of January, 1969 had shown an obstruction of the third portion of the duodenum by a band-like structure (Fig. la). Active peristaltic waves moved barium toward the site of obstruction and then back from it. Hypotonic duodenography (Fig. Ib) also demonstrated these findings but showed no intrinsic abnormality. Radiograms taken at another hospital just before her first visit to UMMC were reviewed by UMMC radiologists and read as normal, as were the IVP, barium enema, and cholecystogram studies. Repeat radiograms on August 25, 1972 showed an entirely normal third portion of the duodenum (Fig. 2). The previously noted dilatation of the third portion of the duodenum was no longer present.
A large number of laboratory studies were done when she was vomiting. All results were normal in studies which included electrolytes, CBC, FBS, BUN, amylase, liver function tests, infectious mononucleosis screen, pregnancy test, stool guaiac, stool for ova and parasites, histoplasmin and tuberculin skin tests, and urine culture. Urinalyses were normal except for intermittent ketonuria and trace albuminuria. Skull and chest films were normal.

Psychiatric evaluation described her as a 13-year-old girl who appeared friendly, smiling, and talked easily to the psychiatrist. Her attitude towards her vomiting was the classic hysterical stance of "la belle indifference." In contrast, however, was her active concern about the physical changes of early adolescence, particularly her breast size. Her parents were on the verge of divorce, with the father's drinking one of the issues. The father had had episodic vomiting for years and the father's vomiting was openly compared to that of the daughter by the family. Her father's relationship to her appeared teasing and provocative. Her onset of vomiting coincided with the mother's declaration that she was leaving the girl and her father at home while she went to visit a relative. This vomiting kept her mother home; in fact, the repeated hospitalizations quieted the parents' talk of divorce and served to keep the family together.

\section{Discussion}

The superior mesenteric artery syndrome (SMAS) consists of the clinical symptoms of lower duodenal obstruction and radiographic 
is emptied, anterior compression of the duodenum at the level of the SMA may not be demonstrable radiographically. Other authors ${ }^{3,6.11}$ also point out that the radiographic findings of SMAS may not be present between attacks.

Radiographic findings similar to those seen in SMAS were seen by Fischer ${ }^{12}$ in association with dilatation of the duodenum from other causes. He pointed out the importance of motor dysfunction in the development of the "large duodenum." Radiographic findings may be secondary to dilatation rather than to an extrinsic obstructive factor such as a band. He included emotional status as a factor influencing duodenal motor activity.

Our patient had clearly recognizable emotional factors. In her final episode of vomiting, the improvement associated with hospitalization was obtained with outpatient treatment, assuring the patient and her family that there was no physical basis for the vomiting. Continued supportive follow-up by one physician was arranged. Psychotherapy was recommended but was not acceptable to the family.

Conservative treatment of the SMAS includes decompression of the stomach, hydration with intravenous fluids, removal of constricting abdominal casts or bandages, and placing the patient in the right lateral position to allow the mesentery to swing away from the posterior abdominal wall. Conservative therapy was emphasized in the literature reviewed. According to some authors, should surgery be indicated, duodenojejunostomy is recommended..$^{2.7 .11}$

\section{References}

1. Von Rokitansky, C.: Lehrbuch der Pathologischen Anatomie, Wien, Braümuller, 1861.

2. Bunch, W. and Delaney, J.: Scoliosis and acute vascular compression of the duodenum. Surgery 67: 901,1970

3. Guthrie, R. H.: Wilkie's syndrome. Ann. Surg. 173: $290,1971$.

4. Wallace, R. G. and Howard, W. B.: Acute superior mesenteric artery syndrome in the severely burned patient. Radiology 94: 307, 1970.

5. Mindell, H. J and Holm, J. L.: Acute superior mesenteric artery syndrome. Radiology 94: 299, 1970.

6. Hyde, J. S., Swarts, C. L., Nicholas, E. E., Snead, C. R. and Strasser, N. F.: Superior mesenteric artery syndrome. Am. J. Dis. Child. 106: 59, 1963.

7. Kaiser, G. C., McKain, J. M. and Schumacker, H. B.: The superior mesenteric artery syndrome. Surg. Gynecol. Obstet. 110: 133, 1960.

8. Barner, H. B., and Sherman, D. C.: Vascular compression of the duodenum. Surg. Gynecol. Obstet. Abstracts 117: 103, 1963.

9. Spiro, H.: Clinical Gastroenterology. New York. McMillan Co., 1970, p. 341.

10. Barnes, G. R. and Gwinn, J. L.: Superior mesenteric artery syndrome. Am. J. Dis. Child. 107: $317,1964$.

11. Goin, L. S, and Wilk, S. P.: Intermittent arteriomesenteric occlusion of the duodenum. Radiology 67: 729, 1956.

12. Fischer, H. W.: The big duodenum. Am. J. Roentgenol. 83: 861,1960 .

\section{Threat of New Influenza B Strains}

Influenza B viruses, which contain hemagglutinins showing marked antigenic changes from the $B$ viruses isolated previously, are being reported in sporadic cases from various parts of the world. This observation raises fears that populations which in the past were exposed or immunized to older known influenza B strains may not be adequately protected against these new strains.

The Center for Disease Control in Atlanta raises questions as to whether any of these strains will become dominant worldwide, and therefore should be incorporated into influenza vaccines. "Hopefully, increased surveillance in countries where the influenza season is now beginning may provide more information upon which to base sound recommendations for vaccine formulation and usage."-From CDC Morbidity and Mortality Weekly Report, May 19, 1973. 